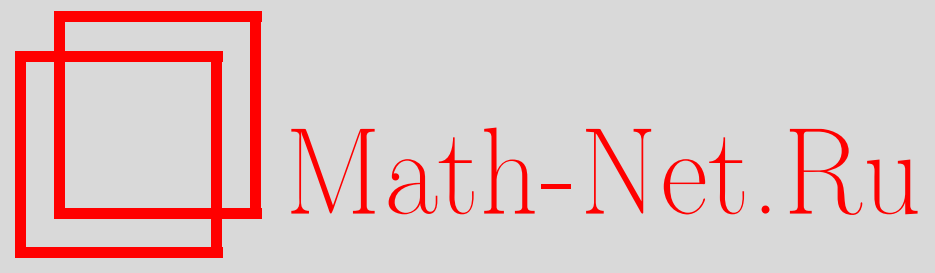

М. В. Долгополов, С. П. Заводов, Е. Ю. Петрова, Бифуркационные наборы расширенного потенциала Хиггса, Вестн. Сам. гос. техн. ун-та. Сер. Физ.-мат. науки, 2013, выпуск 4(), 173-183

DOI: https://doi.org/10.14498/vsgtu1253

Использование Общероссийского математического портала MathNet.Ru подразумевает, что вы прочитали и согласны с пользовательским соглашением

http://www.mathnet.ru/rus/agreement

Параметры загрузки:

IP : 52.87 .193 .239

26 апреля 2023 г., 08:04:24

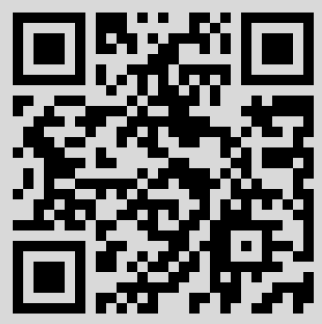




\title{
БИФУРКАЦИОННЫЕ НАБОРЫ РАСШИРЕННОГО ПОТЕНЦИАЛА ХИГГСА
}

\author{
М. В. Долгополов, С. П. Заводов, Е. Ю. Петрова \\ Самарский государственный университет, \\ Россия, 443011, Самара, ул. Академика Павлова, 1. \\ E-mails: mikhaildolgopolov@rambler.ru, zavodov.sp@gmail.com, kuleobul@rambler.ru
}

\begin{abstract}
Одной из наиболее актуальных проблем современной бизики элементарных частиц, является обоснование генерации барионного заряда во Вселенной. В суперсимметричных моделях с расширенным сектором Хиггса возможсно описание бариогенезиса с привлечением теории фазовых переходов и теории катастроф. В работе в рамках минимальной суперсимметрии рассмотрена эволюиия потенциала Хиггса при изменении температуры и управляюших параметров модели. Учтена температурная зависимость самих параметров $\lambda_{1}(T), \ldots, \lambda_{7}(T)$; рассмотрены условия существования устойчивого минимума системы и получены области значений параметров $A, \mu, \operatorname{tg} \beta . B$ пределе нулевой температурь при массе бозона Хиггса $m_{h}=125$ ГэВ теоретические предсказания блестяще согласуются с экспериментальными данными. Получены наборы параметров модели, при которых система претерпевает бифуркаиии.
\end{abstract}

Ключевые слова: бифуркации потенциала Хиггса, эволюиия Вселенной, фазовый переход, базис Гребнера.

Результаты данной работы докладывались на Третьей международной конференции «Математическая физика и её приложения» $(27$ августа - 1 сентября 2012 г., г. Самара)

Введение. На современном этапе развития физики частиц предполагается, что Вселенная заполнена некоторым полем со спином 0, называемым хиггсовым полем, которое является дублетом в $S U(2)$-пространстве и переносит ненулевой $U(1)$-гиперзаряд, а также является синглетом в цветном пространстве. Калибровочные бозоны и фермионы, взаимодействуя с хиггсовским полем, оказываются частицами с ненулевыми массами. При этом важно, что состояния Вселенной с одним или несколькими хиггсовскими полями не ортогональны основному состоянию без них (т.е. вакууму), несмотря на то, что эти состояния имеют ненулевые $S U(2)$ - и $U(1)$-квантовые числа $[1,2]$. Таким образом, считается, что массу частицы приобретают за счёт взаимодействия с самодействующим скалярным полем, распространённым во всём пространстве. Кванты этого поля - бозоны Хиггса.

Стандартная модель (CM) физики элементарных частиц содержит всего один нейтральный скалярный бозон Хиггса. В её рамках получены массы $W^{ \pm}$, $Z^{0}$-бозонов, блестяще согласующиеся с экспериментом, во многих лабораториях мира, в особенности её предсказания были проверены до долей процента в ЦЕРН на LEP со встречными электрон-позитронными пучками. И всё же СМ имеет внутренние трудности. Выделим из них следующие:

Михаил Вячеславович Долгополов (к.ф.-м.н., доц.), доцент, каф. общей и теоретической физики; заведующий лабораторией, научно-исследовательская лаборатория математической физики. Семён Павлович Заводов, лаборант, научно-исследовательская лаборатория математической физики. Елена Юръевна Петрова, младший научный сотрудник, научноисследовательская лаборатория математической физики. 
1) СМ содержит порядка 20-ти свободных параметров (массы частиц, константы взаимодействий, параметры матрицы Кабиббо-КобаяшиМаскава $V_{C K M}$ и др.);

2) в СМ последовательно объединены только электромагнитное и слабое взаимодействия (электрослабое взаимодействие); сильное взаимодействие рассматривается как независимое, а гравитационное вовсе не входит в схему СМ;

3) $\mathrm{CM}$ не даёт ответа на вопросы о происхождении иерархии масс наблюдаемых элементарных частиц, о количестве поколений наблюдаемых фундаментальных фермионов, о размерности нашего пространствавремени, о механизме генерации барионной асимметрии наблюдаемой Вселенной, об отсутствии частиц-кандидатов на роль тёмной материи.

На протяжении более сорока лет из частиц СМ экспериментально не был обнаружен лишь бозон Хиггса. И только в 2012 году каждый из основных детекторов Большого адронного коллайдера - ATLAS и CMS - наблюдал новую частицу с массой около $125 \div 126$ ГэВ, по свойствам напоминающую бозон Хиггса (см., например, [3,4]). Изучение свойств бозона Хиггса и описание бариогенезиса являютя одними из самых актуальных проблем физики частиц на сегодняшний день.

В СМ существуют лишь косвенные ограничения на его массу, возникающие из условий устойчивости хиггсовского потенциала и из требования необращения константы связи в нуль и в бесконечность при энергиях ниже $1 \mathrm{T \ni B} \mathrm{[5].}$

Для разрешения проблем СМ необходимо выйти за её рамки и рассмотреть новую физику при энергиях порядка 100 ГэВ и выше. В этом случае CM можно рассматривать как низкоэнергетическое эффективное приближение более фундаментальной теории, характеризуемой более высоким массовоэнергетическим масштабом.

Многие низкоэнергетические эффективные теории содержат элементарные скаляры, соответствующие неминимальному сектору Хиггса. Одна из наиболее привлекательных теорий - суперсимметрия. В простейших суперсимметричных теориях хиггсовский сектор двухдублетный, вследствие чего появляются три нейтральных и два заряженных бозона Хиггса.

В работе исследуются условия минимума эффективного потенциала при массе лёгкого бозона Хиггса $m_{h}=125$ ГэВ. Построены контурплоты массы бозона Хиггса в пространстве параметров $A, \mu, m_{H^{ \pm}}$.

1. Эффективный потенциал в минимальной суперсимметричной стандартной модели (MCCM). Общая эрмитова форма перенормируемого $S U(2) \times U(1)$ инвариантного потенциала имеет вид

$$
\begin{gathered}
U_{\text {eff }}\left(\Phi_{1}, \Phi_{2}\right)=-\mu_{1}^{2}\left(\Phi_{1}^{+} \Phi_{1}\right)-\mu_{2}^{2}\left(\Phi_{2}^{+} \Phi_{2}\right)-\mu_{12}^{2}\left(\Phi_{1}^{+} \Phi_{2}\right)-\mu_{12}^{2 *}\left(\Phi_{2}^{+} \Phi_{1}\right)+ \\
+\lambda_{1}(T)\left(\Phi_{1}^{+} \Phi_{1}\right)^{2}+\lambda_{2}(T)\left(\Phi_{2}^{+} \Phi_{2}\right)^{2}+\lambda_{3}(T)\left(\Phi_{1}^{+} \Phi_{1}\right)\left(\Phi_{2}^{+} \Phi_{2}\right)+ \\
+\lambda_{4}(T)\left(\Phi_{1}^{+} \Phi_{2}\right)\left(\Phi_{2}^{+} \Phi_{1}\right)+\frac{1}{2} \lambda_{5}(T)\left(\Phi_{1}^{+} \Phi_{2}\right)^{2}+\frac{1}{2} \lambda_{5}^{*}(T)\left(\Phi_{2}^{+} \Phi_{1}\right)^{2}+ \\
+\lambda_{6}(T)\left(\Phi_{1}^{+} \Phi_{1}\right)\left(\Phi_{1}^{+} \Phi_{2}\right)+\lambda_{6}^{*}(T)\left(\Phi_{1}^{+} \Phi_{1}\right)\left(\Phi_{2}^{+} \Phi_{1}\right)+ \\
+\lambda_{7}(T)\left(\Phi_{2}^{+} \Phi_{2}\right)\left(\Phi_{1}^{+} \Phi_{2}\right)+\lambda_{7}^{*}(T)\left(\Phi_{2}^{+} \Phi_{2}\right)\left(\Phi_{2}^{+} \Phi_{1}\right),
\end{gathered}
$$


где дублеты полей можно представить следующим образом:

$$
\begin{gathered}
\Phi_{1}=\left(\begin{array}{c}
\phi_{1}^{+} \\
\phi_{1}^{0}
\end{array}\right)=\left(\begin{array}{l}
-i \omega_{1}^{+} \\
\frac{1}{\sqrt{2}}\left(v_{1}+\eta_{1}+i \chi_{1}\right)
\end{array}\right), \\
\Phi_{2}=\left(\begin{array}{c}
\phi_{2}^{+} \\
\phi_{2}^{0}
\end{array}\right)=\left(\begin{array}{l}
-i \omega_{2}^{+} \\
\frac{1}{\sqrt{2}}\left(v_{2}+\eta_{2}+i \chi_{2}\right)
\end{array}\right) .
\end{gathered}
$$

Вакуумные ожидания этих полей такие:

$$
\Phi_{1}=\frac{1}{\sqrt{2}}\left(\begin{array}{c}
0 \\
v_{1}
\end{array}\right), \quad \Phi_{2}=\frac{1}{\sqrt{2}}\left(\begin{array}{c}
0 \\
v_{2}
\end{array}\right) .
$$

Здесь $v_{1}^{2}+v_{2}^{2}=v^{2}$, а $\mu_{12}^{2}, \lambda_{5}, \lambda_{6}, \lambda_{7}$ могут быть комплексными величинами. В потенциале Хиггса (1) температурная зависимость явно представлена в высокотемпературных поправках к параметрам $\lambda_{1}, \ldots, \lambda_{7}$ [6], которые определяются для эволюции эффективного потенциала граничными условиями на масштабе суперсимметрии $M_{S U S Y}$. Аналитические выражения для параметров $\mu_{1}^{2}, \mu_{2}^{2}$ получены в работе [7].

Согласно работе [8] в статье используется нелинейное преобразование

$$
\lambda_{i}=\lambda_{i}\left(\alpha, \beta, m_{h}, m_{H}, m_{A}, m_{H^{ \pm}} \lambda_{6}, \lambda_{7}\right), \quad i=1,2, \ldots, 5
$$

для масс скаляров и углов смешивания, приводящее в общем случае к базису массовых состояний $\left(h, H\right.$, и $A$ обозначают нейтральные скаляры, $H^{+}, H^{-}-$ заряженные бозоны Хиггса, $\alpha$-угол смешивания $C P$-чётных скаляров $h$ и $\left.H, \operatorname{tg} \beta=v_{2} / v_{1}\right)$.

Общий анализ эффективного потенциала весьма сложен. Условия возникновения физических состояний массивных частиц определяются в нетривиальном минимуме потенциала (1). Для упрощения анализа потенциала (1) перепишем его в системе вакуумных ожиданий:

$$
\begin{aligned}
U_{\text {eff }}\left(v_{1}, v_{2}\right)=-\frac{1}{2} \mu_{1}^{2} v_{1}^{2}-\frac{1}{2} \mu_{2}^{2} v_{2}^{2} & -\operatorname{Re} \mu_{12}^{2} v_{1} v_{2}+\frac{1}{4} \lambda_{1} v_{1}^{4}+\frac{1}{4} \lambda_{2} v_{2}^{4}+ \\
& +\frac{1}{4} \lambda_{345} v_{1}^{2} v_{2}^{2}+\frac{1}{2} \operatorname{Re} \lambda_{6} v_{1}^{3} v_{2}+\frac{1}{2} \operatorname{Re} \lambda_{7} v_{1} v_{2}^{3},
\end{aligned}
$$

где $\lambda_{345}=\lambda_{3}+\lambda_{4}+\operatorname{Re} \lambda_{5}$.

При понижении температуры $T$ от нескольких сотен ГэВ до почти нулевой температуры в ходе космологической эволюции изначально симметричный потенциал (1) с минимумом в точке $\left(v_{1}=0, v_{2}=0\right)$ в пространстве фоновых полей видоизменяется до тех пор, пока в системе не возникает «истинный» минимум, для которого вакуумное ожидание на сегодняшний день $v=\sqrt{v_{1}^{2}+v_{2}^{2}}=246$ ГэВ.

На масштабе масс суперпартнёров всегда выполняются граничные условия $\lambda_{1}=\lambda_{2}=\left(g_{1}^{2}+g_{2}^{2}\right) / 8, \lambda_{3}=\left(g_{2}^{2}-g_{1}^{2}\right) / 4, \lambda_{4}=-g_{2}^{2} / 2, \lambda_{5}=\lambda_{6}=\lambda_{7}=0$.

Экстремум потенциала при нулевой температуре в отсутствие квантовополевых поправок

$$
U_{0}\left(v_{1}, v_{2}\right)=-\left(g_{1}^{2}+g_{2}^{2}\right)\left(v_{1}^{2}-v_{2}^{2}\right)^{2} / 32
$$


не ограничен снизу и имеет два «плоских направления» $v_{1}= \pm v_{2}$ (рис. $\left.1, \mathrm{a}\right)$.

Для определения устойчивого или неустойчивого равновесия системы дальнейшие рассуждения проводятся в рамках теории катастроф $[9,10]$.

Пороговые поправки к граничным условиям при нулевой температуре получены в [5]. Как правило, они приводят к изменению убывающей функции на седловую конфигурацию, слабо возрастающую вдоль одного из плоских направлений и более существенно убывающего вдоль другого из них. При нулевой температуре имеет место плавный переход поверхности минимумов потенциала в седловую конфигурацию с увеличением значения трилинейной константы связи $A=A_{t}=A_{b}$ (рис. 1).

Согласно работе [5] дополнительное условие

$$
\operatorname{Re} \mu_{12}^{2}=s_{\beta} c_{\beta}\left[m_{A}^{2}-\frac{v^{2}}{2}\left(2 \operatorname{Re} \lambda_{5}+\operatorname{Re} \lambda_{6} \operatorname{ctg} \beta+\operatorname{Re} \lambda_{7} \operatorname{tg} \beta\right)\right]
$$

где $s_{\beta}=\sin \beta, c_{\beta}=\cos \beta, m_{A}$ - масса псевдоскалярного бозона Хиггса $A$.

Независимыми параметрами двухдублетного потенциала являются $\operatorname{tg} \beta=$

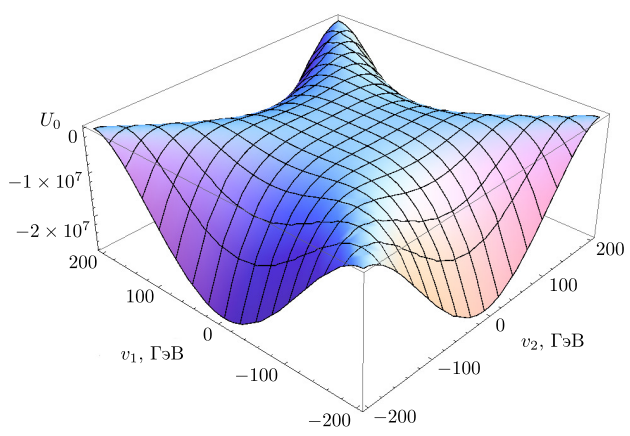

a

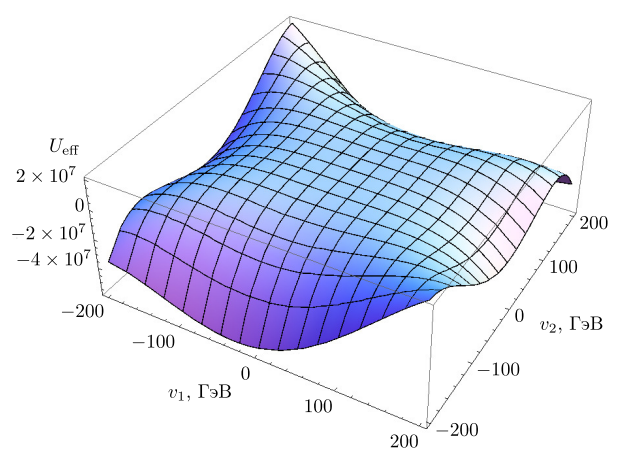

$B$

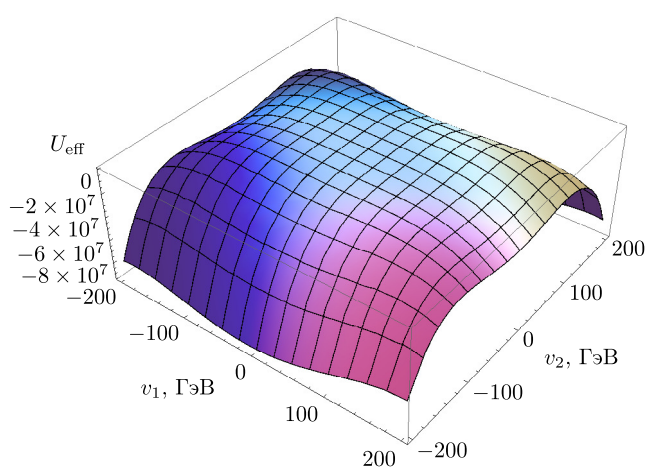

б

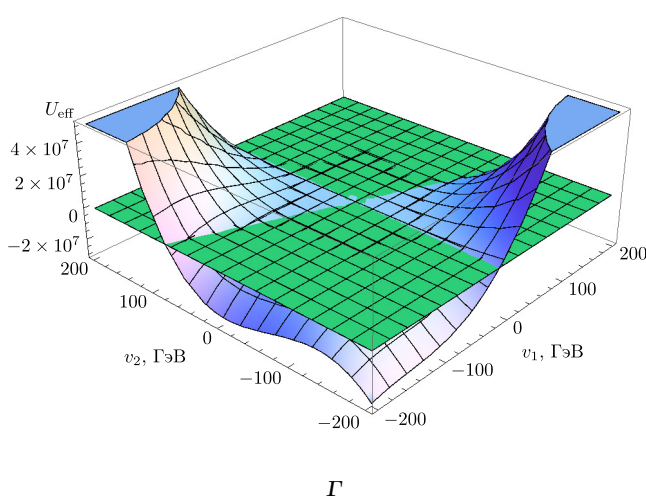

Рис. 1. Поверхности эффективного потенциала $U_{\min }$ в экстремальном состоянии (3) при $T=0:$ a) при отсутствии квантовых поправок за счёт взаимодействия «сектор Хиггса - скварки»; б-г) с учётом квантовых поправок. Для численного расчёта взяты параметры $m_{W}=79,96$ ГэВ, $m_{t}=175 \Gamma_{\ni} \mathrm{B}, M_{S U S Y}=500 \Gamma_{\ni} \mathrm{B}, m_{H^{ \pm}}=150 \Gamma_{\ni} \mathrm{B}, g_{1}=0,3573, g_{2}=0,6517, h_{t}=1, h_{b}=0,1$, $g_{s}=0,1075, v=246$ ГэВ $\operatorname{tg} \beta=5$; параметры суперполя $\mu=500$ ГэВ и $A=1000$ ГэВ (б), $A=1500$ ГэВ (в), $A=2000$ ГэВ (г) 
$=v_{2} / v_{1}$ и масса заряженного скаляра

$$
m_{H^{ \pm}}^{2}=m_{W}^{2}+m_{A}^{2}-\frac{v^{2}}{2}\left(\operatorname{Re} \Delta \lambda_{5}-\Delta \lambda_{4}\right),
$$

где $\Delta \lambda_{i}$ - радиационные поправки к параметрам на масштабе $m_{t}, m_{W}^{2}=$ $=v^{2} g_{2}^{2} / 4$.

2. Связи между параметрами минимальной и неминимальной суперсимметричных (НМССМ) моделей при $\boldsymbol{m}_{\boldsymbol{h}}=125$ ГэВ. В МССМ два нейтральных $C P$-чётных $h$ и $H$, два заряженных $H^{ \pm}$и один псевдоскалярный $A$ бозонов Хиггса, явный вид которых (в СР-сохраняющем пределе) получен в работе [5]:

$$
\begin{gathered}
m_{h}^{2}=s_{\alpha+\beta}^{2} m_{Z}^{2}+c_{\alpha-\beta}^{2} m_{A}^{2}-v^{2}\left(-2 c_{\alpha+\beta}\left(\operatorname{Re} \Delta \lambda_{6} s_{\alpha} c_{\beta}-\operatorname{Re} \Delta \lambda_{7} c_{\alpha} s_{\beta}\right)+\right. \\
\left.+\Delta \lambda_{1} s_{\alpha}^{2} c_{\beta}^{2}+\Delta \lambda_{2} c_{\alpha}^{2} s_{\beta}^{2}-2\left(\Delta \lambda_{3}+\Delta \lambda_{4}\right) c_{\alpha} c_{\beta} s_{\alpha} s_{\beta}+\operatorname{Re} \Delta \lambda_{5}\left(s_{\alpha}^{2} s_{\beta}^{2}+c_{\alpha}^{2} c_{\beta}^{2}\right)\right), \\
m_{H}^{2}=c_{\alpha+\beta}^{2} m_{Z}^{2}+s_{\alpha-\beta}^{2} m_{A}^{2}-v^{2}\left(2 s_{\alpha+\beta}\left(\operatorname{Re} \Delta \lambda_{6} c_{\alpha} c_{\beta}+\operatorname{Re} \Delta \lambda_{7} s_{\alpha} s_{\beta}\right)+\right. \\
\left.+\Delta \lambda_{1} c_{\alpha}^{2} c_{\beta}^{2}+\Delta \lambda_{2} s_{\alpha}^{2} s_{\beta}^{2}+2\left(\Delta \lambda_{3}+\Delta \lambda_{4}\right) c_{\alpha} c_{\beta} s_{\alpha} s_{\beta}+\operatorname{Re} \Delta \lambda_{5}\left(c_{\alpha}^{2} s_{\beta}^{2}+s_{\alpha}^{2} c_{\beta}^{2}\right)\right), \\
m_{H^{ \pm}}^{2}=m_{W}^{2}+m_{A}^{2}-\frac{v^{2}}{2}\left(\operatorname{Re} \Delta \lambda_{5}-\Delta \lambda_{4}\right),
\end{gathered}
$$

где $\Delta \lambda_{i}, i=1,2, \ldots, 7$ - радиационные поправки к параметрам на масштабе массы топ-кварка $m_{t} ; \alpha$-угол смешивания, определяемый из выражения

$$
\operatorname{tg}(2 \alpha)=\frac{s_{2 \beta}\left(m_{A}^{2}+m_{Z}^{2}\right)+v^{2}\left(\left(\Delta \lambda_{3}+\Delta \lambda_{4}\right) s_{2 \beta}+2 c_{\beta}^{2} \operatorname{Re} \Delta \lambda_{6}+2 s_{\beta}^{2} \operatorname{Re} \Delta \lambda_{7}\right)}{c_{2 \beta}\left(m_{A}^{2}-m_{Z}^{2}\right)+v^{2}\left(\Delta \lambda_{1} c_{\beta}^{2}-\Delta \lambda_{2} s_{\beta}^{2}-\operatorname{Re} \Delta \lambda_{5} c_{2 \beta}+s_{2 \beta}\left(\operatorname{Re} \Delta \lambda_{6}-\operatorname{Re} \Delta \lambda_{7}\right)\right)},
$$

$\beta=\operatorname{arctg}\left(v_{2} / v_{1}\right) ; m_{W}, m_{Z}-$ массы $W$ - и $Z$-бозонов.

Согласно современным экспериментальным данным масса нейтрального $C P$-чётного бозона Хиггса с нулевым спином равна 125 ГэВ. В МССМ легчайшим бозоном Хиггса, удовлетворяющим этим условиям, является $m_{h}$, масса которого определена выражением (4). Считая значение $m_{h}$ заданным, из соотношений (4), (5), (6) можно получить набор параметров модели, описывающих экспериментальную картину.

В НMCСМ после диагонализации массовой матрицы в минимуме определяется спектр бозонов Хиггса, состоящий из трёх $C P$-чётных, двух $C P$ нечётных и двух заряженных бозонов Хиггса:

а) CP-чётные бозоны Хиггса:

$$
\begin{gathered}
m_{H_{1}}^{2}=2 \sqrt{(-q)} \cos \left(\frac{\Theta+2 \pi}{3}\right)-\frac{a_{2}}{3}, m_{H_{2}}^{2}=2 \sqrt{(-q)} \cos \left(\frac{\Theta-2 \pi}{3}\right)-\frac{a_{2}}{3}, \\
m_{H_{3}}^{2}=2 \sqrt{(-q)} \cos \left(\frac{\Theta}{3}\right)-\frac{a_{2}}{3}
\end{gathered}
$$

где

$$
\Theta=\arccos \frac{r}{\sqrt{\left(-q^{3}\right)}}, \quad r=\frac{1}{54}\left(9 a_{1} a_{2}-27 a_{0}-2 a_{2}^{3}\right), \quad q=\frac{1}{9}\left(3 a_{1}-a_{2}^{2}\right),
$$




$$
\begin{aligned}
& a_{0}=m_{13}^{2} m_{22}+m_{23}^{2} m_{12}^{2} m_{33}-m_{11} m_{22} m_{33}-2 m_{12} m_{13} m_{23} \text {, } \\
& a_{1}=m_{11} m_{22}+m_{11} m_{33}+m_{22} m_{33}-m_{12}^{2}-m_{13}^{2}-m_{23}^{2} \text {, } \\
& a_{2}=-m_{11}-m_{22}-m_{33}, \\
& m_{11}=\lambda_{1} v^{2} \cos ^{2} \beta-\left(k_{3} v_{3}+k_{5}\right) v_{3} \operatorname{tg} \beta \text {, } \\
& m_{22}=\lambda_{2} v^{2} \sin ^{2} \beta-\left(k_{3} v_{3}+k_{5}\right) v_{3} \operatorname{ctg} \beta \text {, } \\
& m_{33}=8 k_{4} v_{3}^{2}-\frac{k_{5} v^{2} \cos \beta \sin \beta}{v_{3}}+6 k_{6} v_{3} \text {, } \\
& m_{12}=k_{3} v_{3}^{2}+k_{5} v_{3}+v^{2}\left(\lambda_{3}+\lambda_{4}\right) \cos \beta \sin \beta, \\
& m_{13}=v\left(2 k_{1} v_{3} \cos \beta+\left(2 k_{3} v_{3}+k_{5}\right) \sin \beta\right), \\
& m_{23}=v\left(2 k_{2} v_{3} \sin \beta+\left(2 k_{3} v_{3}+k_{5}\right) \cos \beta\right) \text {, } \\
& M^{2}=\frac{1}{2}\left(\begin{array}{lll}
m_{11} & m_{12} & m_{13} \\
m_{21} & m_{22} & m_{23} \\
m_{31} & m_{32} & m_{33}
\end{array}\right) \text {; }
\end{aligned}
$$

б) физические $C P$-нечётные бозоны Хиггса:

$$
m_{A_{1,2}}=\frac{1}{2}\left(n_{11}+n_{22} \pm \sqrt{\left(n_{11}-n_{22}\right)^{2}+4 n_{12}^{2}}\right)
$$

где

$$
\begin{gathered}
n_{11}=-2 v_{3} \operatorname{cosec} 2 \beta\left(k_{3} v_{3}+k_{5}\right), \quad n_{12}=v\left(2 k_{3} v_{3}-k_{5}\right), \\
n_{22}=-2 k_{3} v^{2} \sin 2 \beta-k_{5} \frac{v^{2}}{2 v_{3}} \sin 2 \beta-18 k_{6} v_{3} .
\end{gathered}
$$

На рис. 2 изображены поверхности в пространстве параметров минимальной и неминимальной суперсимметричных моделей постоянной массы легчайшего $C P$-чётного бозона Хиггса, равной 125 ГэВ.

3. Состояния равновесия и бифуркации. В работе развит подход, обсуждавшийся в литературе [6,11]. Предлагаемая статья рассматривает предельно обобщенный случай, когда все параметры $\mu_{1}, \mu_{2}, \mu_{12}, \lambda_{1}, \ldots, \lambda_{7}$ системыненулевые.

Если рассматриваемая физическая система находится в состоянии равновесия (устойчивого или неустойчивого), то $\nabla U_{\text {eff }}=0$. Этому выражению удовлетворят множество точек $\{u\}$, называемое поверхностью равновесия или многообразием катастрофы. При этом тип равновесия определяется собственными значениями матрицы устойчивости, или гессиана, $U_{i j}=\partial^{2} U_{\text {eff }} / \partial v_{i} \partial v_{j}$.

1. Если $\left.\operatorname{det} U_{i j}\right|_{\{u\}} \neq 0$, то в некоторой окрестности этой невырожденной критической точки $u$ всегда можно подобрать локальную систему координат, в которой эта функция может быть представлена морсовским l-седлом (лемма Морса)

$$
\tilde{U}_{\mathrm{eff}}=\tilde{v}_{n-l}^{2}-\tilde{v}_{l}^{2}
$$

где $n, l=1,2, \tilde{v}_{1}, \tilde{v}_{2}$ - переменные в новой системе координат. В случае $l=0$ критическая точка $u$ является точкой устойчивого минимума, $l=n-$ точкой максимума, $l \neq n-$ седловой точкой. При этом 


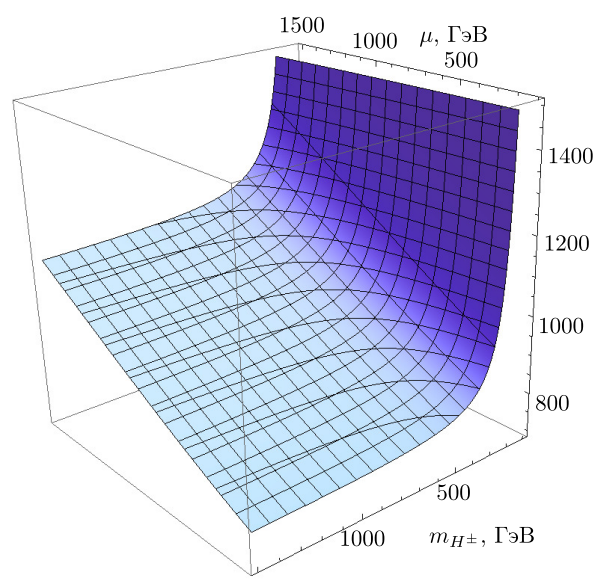

a

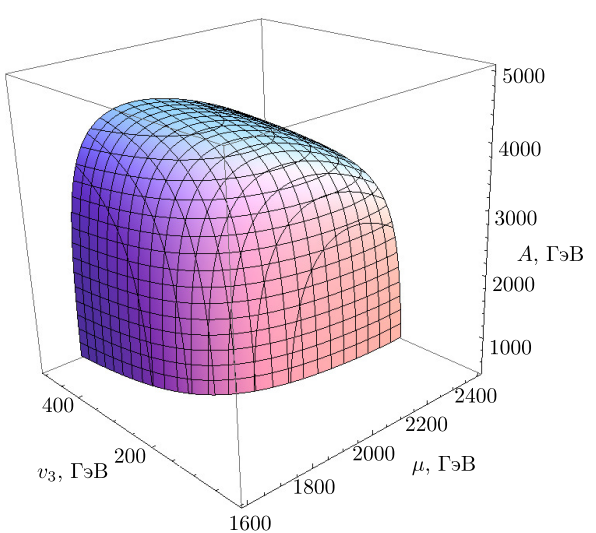

$\sigma$

Рис. 2. Поверхность постоянной массы бозона Хиггса (125 ГэВ) в пространстве параметров $A, \mu, m_{H^{ \pm}}$: а) случай MCСМ; б) случай $\mathrm{HMCCM}\left(v_{1}=v_{2}=v / \sqrt{1+\operatorname{tg}^{2} \beta}, k_{1}=k_{2}=k_{4}=0,6\right.$, $\left.k_{3}=k_{5}=k_{6}=-0,6\right) ; M=1000$ ГэВ, $\operatorname{tg} \beta=5$

$\tilde{U}_{\text {eff }}\left(\tilde{v}_{1}\left(v_{1}, v_{2}\right), \tilde{v}_{2}\left(v_{1}, v_{2}\right)\right)=U_{\text {eff }}\left(v_{1}, v_{2}\right)$, т. е. один и тот же физический процесс может быть описан двумя различными функциями в разных системах координат, а функции $\tilde{U}_{\text {eff }}$ и $U_{\text {eff }}$ являются качественно подобными.

2. Если $\left.\operatorname{det} U_{i j}\right|_{\{u\}}=0$, то в некоторой окрестности вырожденной критической точки $u$ гладкой функции всегда можно подобрать локальную систему координат, в которой эта функция может быть представлена в виде суммы морсовского l-седла и неморсовской части.

В нашем случае потенциал, записанный в новой системе координат, уже не будет физичным в том смысле, что коэффициенты, стоящие перед членами второй степени по полю не являются массовыми членами, однако вид преобразованного потенциала позволит однозначно судить, является ли минимум устойчивым или неустойчивым (см. рис. 3).

Существование критических точек потенциала (3) накладывает условия

$$
\begin{aligned}
& -\mu_{1}^{2} v_{1}-\operatorname{Re} \mu_{12}^{2} v_{2}+\lambda_{1} v_{1}^{3}+\frac{1}{2} \lambda_{345} v_{1} v_{2}^{2}+\frac{3}{2} \operatorname{Re} \lambda_{6} v_{1}^{2} v_{2}+\frac{1}{2} \operatorname{Re} \lambda_{7} v_{2}^{3}=0 \\
& -\mu_{2}^{2} v_{2}-\operatorname{Re} \mu_{12}^{2} v_{1}+\lambda_{2} v_{2}^{3}+\frac{1}{2} \lambda_{345} v_{1}^{2} v_{2}+\frac{1}{2} \operatorname{Re} \lambda_{6} v_{1}^{3}+\frac{3}{2} \operatorname{Re} \lambda_{7} v_{1} v_{2}^{2}=0 .
\end{aligned}
$$

При рассмотрении выделенных направлений фоновых полей возникают бифуркационные множества. Рассмотрим эти бифуркационные наборы:

1) $v_{1}=0, v_{2}=0$ : система находится в устойчивом минимуме, существовавшем при высоких температурах до возникновения фазового перехода;

2) а) $v_{1} \rightarrow 0, v_{2} \neq 0$ : после корректного выбора условий возникновения критических точек (из условий (7), (8) ограничения налагаются на $\mu_{2}^{2}$ и $\left.\operatorname{Re} \mu_{12}^{2}\right)$, гессиан системы имеет вид

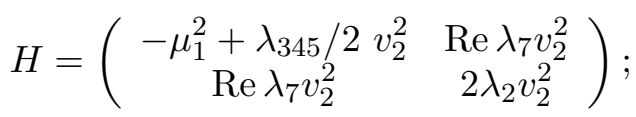




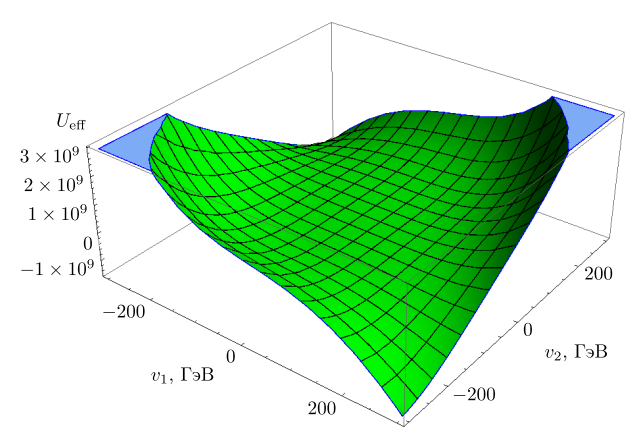

a

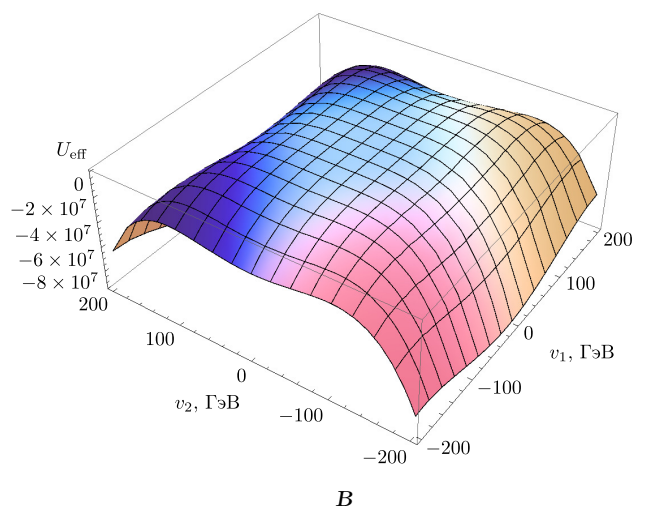

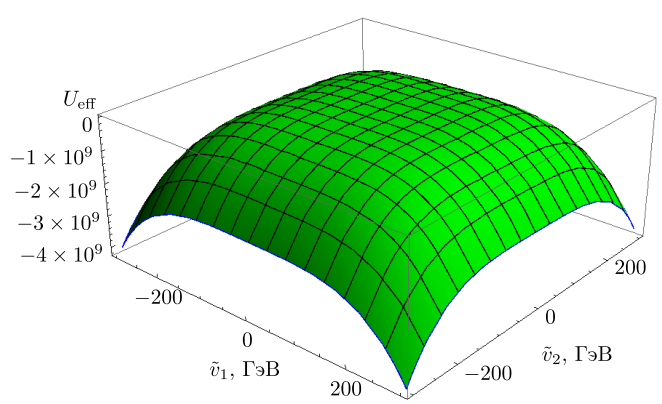

$\sigma$

Рис. 3. Поверхности потенциала Хиггса (3) в точке экстремума в общем случае $(\mathrm{a}, \sigma)$ и в «истинном» минимуме при $T=0, m_{h}=$ $=125$ ГэВ (в): а) старая система координат; б) локальная система координат в окрестности невырожденной критической точки; в) расчёт при следующих параметрах MCCM: $m_{Q}=$ $=500 \Gamma \ni \mathrm{B}, m_{U}=200 \Gamma \ni \mathrm{B}, m_{D}=800$ Гэ $\mathrm{B}, A=$ $=950 \Gamma \ni \mathrm{B}, \mu=750$ ГэВ,$M_{S U S Y}=1$ ТэB

б) $v_{1} \neq 0, v_{2} \rightarrow 0:$ из условий $(7),(8)$ ограничения налагаются на параметры $\mu_{1}^{2}$ и $\operatorname{Re} \mu_{12}^{2}$,

$$
H=\left(\begin{array}{cc}
2 \lambda_{1} v_{1}^{2} & \operatorname{Re} \lambda_{6} v_{1}^{2} \\
\operatorname{Re} \lambda_{6} v_{1}^{2} & -\mu_{2}^{2}+\lambda_{345} / 2 v_{1}^{2}
\end{array}\right) ;
$$

численный анализ показал, что при любых допустимых на сегодняшний день теоретических ограничениях на параметры $A$ и $\mu$ потенциальная функция (3) находится в максимуме, фазовый переход при этом не происходит;

3) $v_{1} \neq 0, v_{2} \neq 0$ : из условий $(7),(8)$ ограничения налагаются на параметры $\mu_{1}^{2}$ и $\mu_{2}^{2}$,

$$
H=\frac{1}{2}\left(\begin{array}{ll}
H_{11} & H_{12} \\
H_{21} & H_{22}
\end{array}\right)
$$

где

$$
\begin{gathered}
H_{11}=\left(2 \operatorname{Re} \mu_{12}^{2} v_{2}+4 \lambda_{1} v_{1}^{3}+3 \operatorname{Re} \lambda_{6} v_{1}^{2} v_{2}-\operatorname{Re} \lambda_{7} v_{2}^{3}\right) / v_{1}, \\
H_{12}=H_{21}=-2 \operatorname{Re} \mu_{12}^{2}+2 \lambda_{345} v_{1} v_{2}+3 \operatorname{Re} \lambda_{6} v_{1}^{2}+3 \operatorname{Re} \lambda_{7} v_{2}^{2}, \\
H_{22}=\left(2 \operatorname{Re} \mu_{12}^{2} v_{1}+4 \lambda_{2} v_{2}^{3}-\operatorname{Re} \lambda_{6} v_{1}^{3}+3 \operatorname{Re} \lambda_{7} v_{1} v_{2}^{2}\right) / v_{2} .
\end{gathered}
$$

Таким образом, система (3) находится в устойчивом минимуме, если $\operatorname{det} H>0$ и $\operatorname{Tr} H>0$ (см. рис. 4$)$. 
При увеличении значений параметров до $A \sim 2000$ ГэВ, $\mu \sim 2000$ ГэВ устойчивого минимума не существует.

Обычно, вычисляя стационарные точки потенциала, мы приходим к нелинейной, неоднородной системе уравнений, что усложняет задачу. Однако мы можем определить многие свойства минимума потенциала Хиггса, не решая при этом нелинейную систему. Можно упростить, ускорить и автоматизировать анализ общего потенциала Хиггса с помощью т. н. базиса Грёбнера, современного алгебраического подхода для определения глобального минимума [12]. Применяя метод Грёбнера, авторы вычислили все стационарные точки, при которых наименьшее значение потенциала может быть идентифицировано как глобальный минимум.
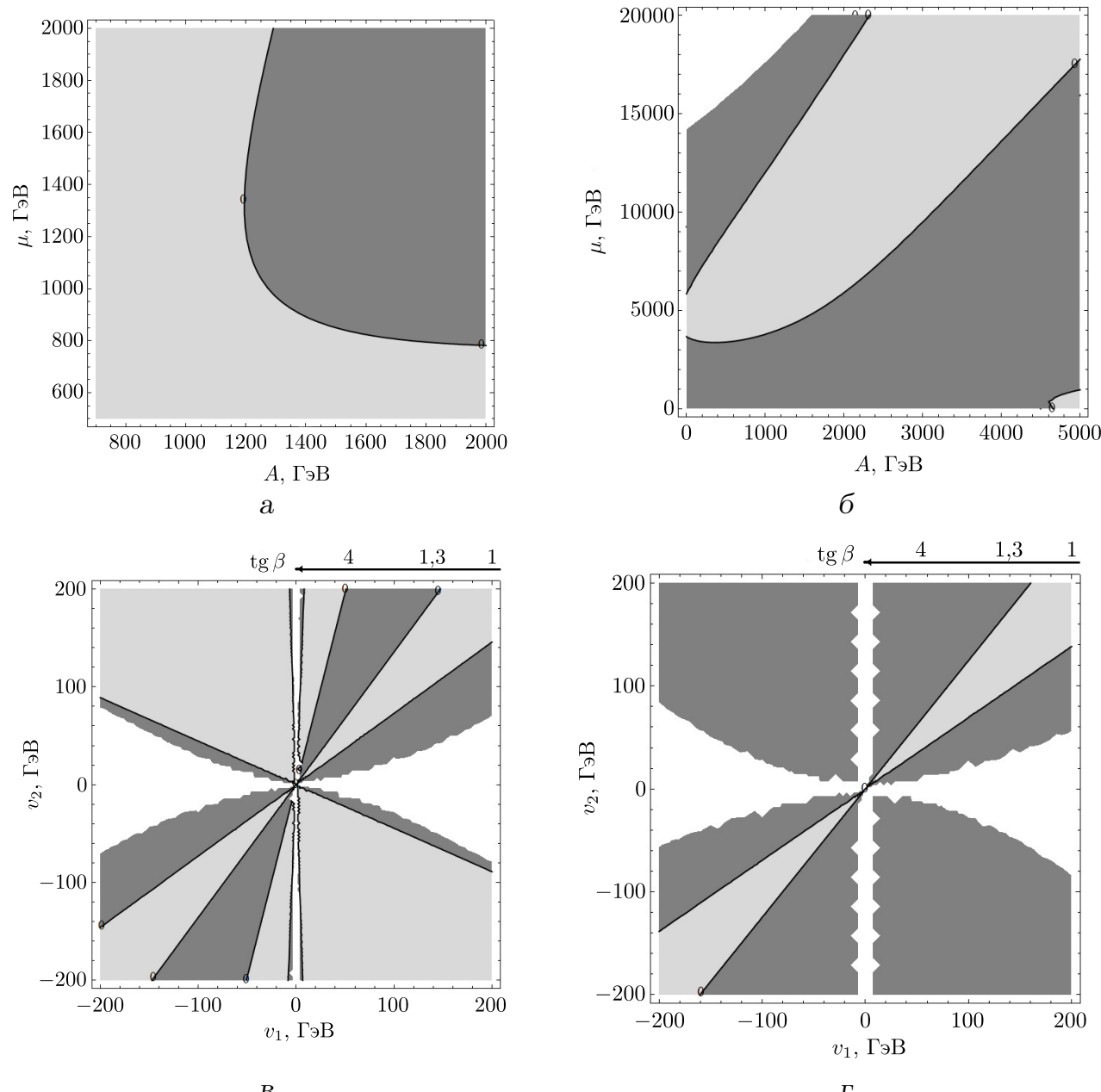

B

Рис. 4. Поведение потенциальной функции при $v_{1} \neq 0, v_{2} \neq 0$ в пространстве параметров $(A, \mu)(\mathrm{a}, \sigma)$ и вакуумных ожиданий $\left(v_{1}, v_{2}\right)(в, \Gamma):$ a) $\operatorname{tg} \beta=5, T=120$ ГэВ; б) $\operatorname{tg} \beta=5$, $T=150$ ГэВ; в) $A=1200$ ГэВ $\mu=950$ ГэВ; г) $A=2000$ ГэВ $\mu=1500$ ГэВ. Светло-серая область соответствует условию $\operatorname{det} H>0$, серая $-\operatorname{det} H<0$, чёрная сплошная линия $-\operatorname{det} H=0$, $\operatorname{Tr} H>0$. Здесь взяты следующие значения параметров для суперпартнёров: $m_{Q}=1100$ ГэВ, $m_{U}=$ $=1200$ ГэВ, $m_{D}=1300$ ГэВ, $m_{H^{ \pm}}=150$ ГэВ, $m_{W_{T_{0}}}=79,96$ ГэВ, $m_{W}=\sqrt{m_{W_{t_{0}}}^{2}+5 g_{2}^{2} T^{2} / 2}, m_{Z}=$ $=\sqrt{\left(g_{1}^{2}+g_{2}^{2}\right) m_{W}^{2} / g_{2}^{2}}, h_{t}=\sqrt{2} m_{t} /(v \sin \beta), h_{b}=\sqrt{2} m b /(v \cos \beta), v=T$ (критерий Шапошникова) 
Заключение. В работе рассмотрен подход к исследованию эффективного потенциала двухдублетной модели физики частиц, позволяющий однозначно устанавливать наборы параметров $\operatorname{MCCM}(A, \mu, \operatorname{tg} \beta)$, при которых потенциал Хиггса находится в состоянии устойчивого и неустойчивого равновесия, а также претерпевает бифуркационные изменения.

Проведено исследование потенциала Хиггса при нулевой температуре. Показано, что в отсутствии квантовых поправок к параметрам $\lambda_{1}, \ldots, \lambda_{7}$ устойчивого минимума системы не возникает; при учёте поправок критической точкой является седло, наиболее выраженное при рассмотрении случая ненулевой температуры.

Рассмотрены поверхности равновесия в отсутствие критических точек и в окрестности изолированной критической точки. При массе легчайшего $C P$-чётного бозона Хиггса $m_{h}$, равной 125 ГэВ, наблюдается состояние устойчивого минимума системы.

При наиболее вероятной температуре фазового перехода $T \sim 120$ ГэВ построены бифуркационные контурплоты управляющих параметров модели, при которых состояние системы изменяется скачком. Немаловажным результатом является ограничение на параметры модели $A, \mu$ сверху. Для обеспечения условий существования устойчивого минимума последние не могут превышать величины, примерно равной 2 ТэВ. Отношение вакуумных ожиданий $\operatorname{tg} \beta$ при этом изменяется в диапазоне от 4 до 30; также возможны значения $\operatorname{tg} \beta \sim 1$, однако при незначительном увеличении параметров $A, \mu$ ограничение исчезает.

Обычно, вычисляя стационарные точки потенциала, мы приходим к нелинейной неоднородной системе уравнений, что усложняет задачу. Однако можно определить многие свойства минимума потенциала Хиггса, не решая при этом нелинейную систему. Применение базиса Грёбнера позволяет существенно упростить, ускорить и автоматизировать анализ общего потенциала Хиггса.

Авторы благодарят М. Н. Дубинина за идейную постановку задачи, обсуждения и замечания. Исследование выполнено в рамках проекта КИАС РФФИ № 12-02-31795-мол_а, Соглашения ФЦП 1299 и поддержано госзаказом СамГУ 909.2011. Также работа Е. Ю. Петровой частично поддержана грантом РФФИ 11-01-00894-а.

\section{БИБЛИОГРАФИЧЕСКИЙ СПИСОК}

1. G. Kane, Modern Elementary Particle Physics. New York, Amsterdam, Madrid, Paris: Addison-Wesley Publ. Co., 1993. xv+352 pp.; русск. пер.: Г. Кейн, Современная физика элементарных частиц. М.: Мир, 1990. 360 с.

2. M. Peskin, D. Schroeder, An introduction to quantum field theory. New York, Amsterdam, Madrid, Paris: Addison-Wesley Publ. Co., 1995. хxii+842 pp.; русск. пер.: М. Пескин, Д. Шрёдер, Введение в квантовую теорию поля. Ижевск: НИЦ РХД, 2001. 784 с.

3. CMS Collaboration, "Observation of a new boson at a mass of $125 \mathrm{GeV}$ with the CMS experiment at the LHC"// Phys. Lett. B, 2012. T. 716, №1. C. 30-61.

4. ATLAS Collaboration, "Observation of a new particle in the search for the Standard Model Higgs boson with the ATLAS detector at the LHC" // Phys. Lett. B, 2012. № 1. C. 1-29.

5. Э. Н. Ахметзянова, М. В. Долгополов, М. Н. Дубинин, "Нарушение СР-инвариантности в двухдублетном хиггсовском секторе МССМ"// ЭЧАЯ, 2006. Т. 37, №5. C. 1285-1382; англ. пер.: E. N. Akhmetzyanova, M. V. Dolgopolov, M. N. Dubinin, "Violation of CP invariance in the two-doublet higgs sector of the MSSM" // Phys. Part. Nuclei, 2006. Vol. 37, no. 5. Pp. 677-734.

6. M. Dolgopolov, M. Dubinin, E. Rykova, "Threshold corrections to the MSSM finite-temperature Higgs potential" // J. Mod. Phys., 2011. Vol. 2. Pp. 301-322, arXiv: 0901.0524 [hep-ph]. 
7. A. Borisov, M. Dolgopolov, M. Dubinin, Self-energy corrections to the MSSM finite-temperature Higgs potential: PoS (QFTHEP2011) 052.

8. E. Akhmetzyanova, M. Dolgopolov, M. Dubinin, "Higgs bosons in the two-doublet model with CP violation" // Phys. Rev. D, 2005. Vol.71, no.7, 075008. 24 pp., arXiv: hep$\mathrm{ph} / 0405264$.

9. Р. Гилмор, Прикладная теория катастроф. Т.1. М.: Мир, 1984. 350 с. [R. Gilmore, Catastrophe theory for scientists and engineers. Vol. 1. Moscow: Mir, 1984. 350 pp.]

10. Т. Постон, И. Стюарт, Теория катастроф и её приложения. М.: Мир, 1980. 608 с. [T. Poston, I. Stewart, Catastrophe theory and its applications. Moscow: Mir, 1980. 608 pp.]

11. M. Dolgopolov, M. Dubinin, I. Erofeev, E. Rykova, Threshold corrections to the MSSM effective Higgs potential: gaugino and higgsino contributions: PoS (QFTHEP2011) 068.

12. И. В. Аржанцев, Базисы Грёбнера и системы алгебраических уравнений. М.: МЦНМО, 2003. 68 c. [I. V. Arzhantsev, Groebner Bases and Systems of Algebraic Equations. MCCME, 2003. 68 pp.]

Поступила в редакцию $01 / \mathrm{II} / 2013$;

в окончательном варианте - 23/VIII/2013.

MSC: $81 T 10$

\title{
BIFURCATION SETS OF EXTENDED HIGGS POTENTIAL
}

\author{
M. V. Dolgopolov, S. P. Zavodov, E. Yu. Petrova
}

Samara State University,

1, Academician Pavlov st., Samara, 443011, Russia.

E-mails: mikhaildolgopolov@rambler.ru, zavodov.sp@gmail.com, kuleobul@rambler.ru

One of the most actual problems in modern particle physics is the problem of the baryon charge evidence in the Universe. In the frameworks of supersymmetric models, phase transitions and catastrophe theory it is possible to describe the baryogenesis. We explored the temperature evolution of Higgs potential with control parameters in the framework of the MSSM, considered the stable minimum conditions and evaluated the area of constrained parameters $A, \mu, \operatorname{tg} \beta$. The sets of model parameters at which the system undergoes a bifurcation are obtained.

Keywords: bifurcation sets of Higgs potential, Universe evolution, phase transition, Groebner basis.

Original article submitted 01/II/2013; revision submitted 23/VIII/2013.

Mikhail V. Dolgopolov (Ph. D. Phys. \& Math.), Associate Professor, Dept. of General \& Theoretical Physics; Head of the Laboratory, Scientific Research Laboratory of Mathematical Physics. Semen P. Zavodov, Laboratory Technician, Scientific Research Laboratory of Mathematical Physics. Elena Yu. Petrova, Junior Researcher, Scientific Research Laboratory of Mathematical Physics. 